\title{
WAVE1 mediates suppression of phagocytosis by phospholipid-derived DAMPs
}

\author{
Ulrich Matt,,1,2 Omar Sharif,1,2 Rui Martins,, ${ }^{1,2}$ Tanja Furtner, ${ }^{1,2}$ Lorene Langeberg, ${ }^{3}$ Riem Gawish, ${ }^{1,2}$ \\ Immanuel Elbau, ${ }^{1,2}$ Ana Zivkovic, ${ }^{1,2}$ Karin Lakovits, ${ }^{2}$ Olga Oskolkova, ${ }^{4}$ Bianca Doninger, ${ }^{1,2}$ \\ Andreas Vychytil, ${ }^{5}$ Thomas Perkmann, ${ }^{6}$ Gernot Schabbauer, ${ }^{4}$ Christoph J. Binder, ${ }^{1,6}$ \\ Valery N. Bochkov, ${ }^{4}$ John D. Scott, ${ }^{3}$ and Sylvia Knapp ${ }^{1,2}$

\begin{abstract}
${ }^{1}$ Research Center for Molecular Medicine (CeMM) of the Austrian Academy of Sciences, Vienna, Austria. 2Department of Medicine I, Laboratory of Infection Biology, Medical University Vienna, Vienna, Austria. ${ }^{3}$ Howard Hughes Medical Institute, Department of Pharmacology, University of Washington School of Medicine, Seattle, Washington, USA. ${ }^{4}$ Institute of Vascular Biology and Thrombosis Research, Center for Biomolecular Medicine and Pharmacology, ${ }^{5}$ Department of Medicine III, Division of Nephrology and Dialysis, and ${ }^{6}$ Department of Medical and Chemical Laboratory Diagnostics, Medical University Vienna, Vienna, Austria.
\end{abstract}

\begin{abstract}
Clearance of invading pathogens is essential to preventing overwhelming inflammation and sepsis that are symptomatic of bacterial peritonitis. Macrophages participate in this innate immune response by engulfing and digesting pathogens, a process called phagocytosis. Oxidized phospholipids (OxPL) are dangerassociated molecular patterns (DAMPs) generated in response to infection that can prevent the phagocytic clearance of bacteria. We investigated the mechanism underlying OxPL action in macrophages. Exposure to OxPL induced alterations in actin polymerization, resulting in spreading of peritoneal macrophages and diminished uptake of $E$. coli. Pharmacological and cell-based studies showed that an anchored pool of PKA mediates the effects of OxPL. Gene silencing approaches identified the A-kinase anchoring protein (AKAP) WAVE1 as an effector of OxPL action in vitro. Chimeric Wave1 ${ }^{-/-}$mice survived significantly longer after infection with $E$. coli and OxPL treatment in vivo. Moreover, we found that endogenously generated OxPL in human peritoneal dialysis fluid from end-stage renal failure patients inhibited phagocytosis via WAVE1. Collectively, these data uncover an unanticipated role for WAVE1 as a critical modulator of the innate immune response to severe bacterial infections.
\end{abstract}

\section{Introduction}

Invasion of bacteria into the peritoneal cavity leads to the immediate initiation of an inflammatory response. Integral to this response are oxygen radicals that are primarily generated to kill microbes. However, these agents also damage host structures through the peroxidation of membrane phospholipids (1). Oxidized phospholipids (OxPL) are endogenous modulators of the inflammatory response that were recently classified as a new entity of danger-associated molecular patterns (DAMPs) (2). As such, previous reports documented a role for these DAMPs in various inflammatory conditions such as atherosclerosis $(3,4)$ lung inflammation $(5-8)$, or inflammatory brain lesions $(9,10)$. The precise contribution of OxPL to these diseases is not fully understood, with some reports postulating a proinflammatory role (7), while others describe antiinflammatory properties (11). The impact of OxPL on the course of infectious diseases was unknown until we discovered that administration of OxPL impaired survival during E. coli peritonitis by inhibiting phagocytosis of bacteria (12). More recently, OxPL were found involved in the host defense against Mycobacterium leprae in humans, which further underscores the critical crosstalk between innate immunity and lipid metabolism (13).

Phagocytosis of pathogens is a major defense mechanism provided by macrophages and neutrophils. Local control of bacterial replication is a prerequisite to preventing systemic spread and

Authorship note: Ulrich Matt and Omar Sharif contributed equally to this work. Conflict of interest: The authors have declared that no conflict of interest exists. Citation for this article: J Clin Invest. 2013;123(7):3014-3024. doi:10.1172/JCI60681. sepsis (14-16). Mechanistically, phagocytosis is a complex process employing a plethora of receptors and pathways that culminates in the modulation of the actin cytoskeleton (17). Here, we investigated the mechanism of action underlying the detrimental effects of OxPL during E. coli peritonitis. We found that OxPL induce alterations in actin polymerization, which resulted in spreading of peritoneal macrophages and concomitantly diminished uptake of E. coli. Biochemical and gene silencing studies revealed that an anchored pool of PKA mediated these effects. The specificity of PKA activity was provided by the WiskottAldrich syndrome protein (WASP) family verprolin-homologous protein 1 (WAVE1), a protein that controls actin polymerization via the Arp $2 / 3$ complex (18). In contrast to all other WASP family members, WAVE1 is predominantly known as an A-kinase anchoring protein (AKAP) that contributes to the specificity of PKA by tethering it to Arp2/3 (19). Only recently, WAVE1 was found expressed in bone marrow-derived macrophages, but its function there remained unknown (20).

\section{Results}

$O X P L$ as generated during E. coli peritonitis inbibit phagocytosis and induce a cell spread in peritoneal macrophages. We have shown that administration of OxPL impaired survival during E. coli peritonitis by inhibiting phagocytosis of the bacteria (12). Further investigation of this phenomenon has uncovered a role for endogenously produced OxPL as biologically relevant modulators of E. coli infections during peritonitis. Levels of OxPL in the peritoneal lavage fluid (PLF) were significantly increased after infection with $E$. coli when compared with samples from healthy mice, as 
A OxPL level in peritonitis

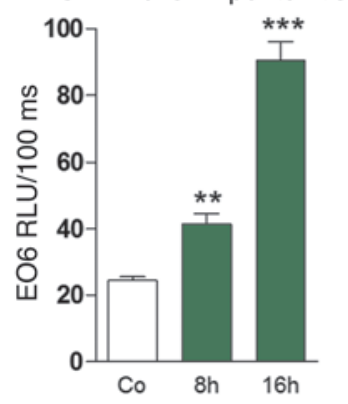

C

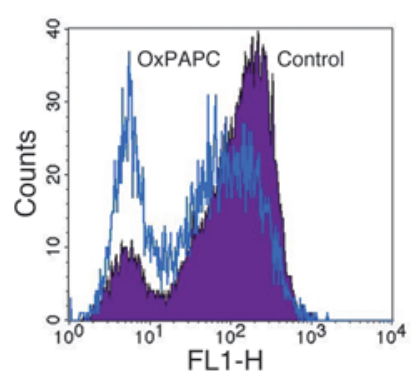

OxPL applied in vitro

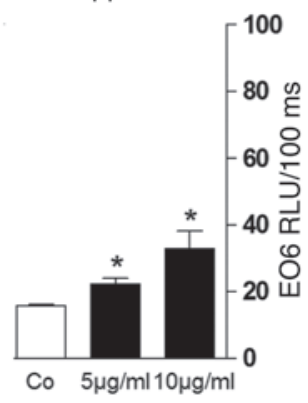

D

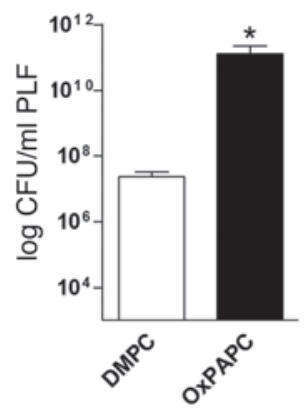

B

E. coli
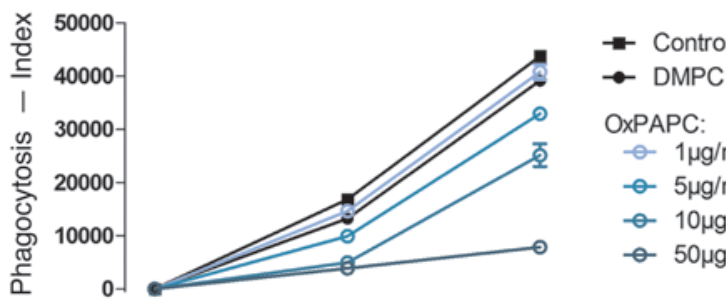

OXPAPC:

๑- $1 \mu \mathrm{g} / \mathrm{ml}$

$\rightarrow 5 \mu \mathrm{g} / \mathrm{ml}$

- $10 \mu \mathrm{g} / \mathrm{m}$

- $50 \mu \mathrm{g} / \mathrm{ml}$

$\mathbf{E}$

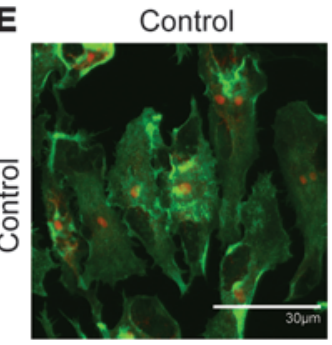

DMPC 120

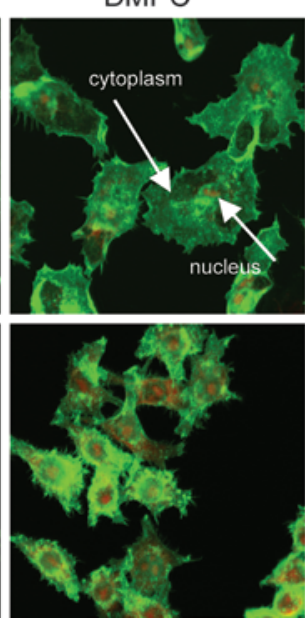

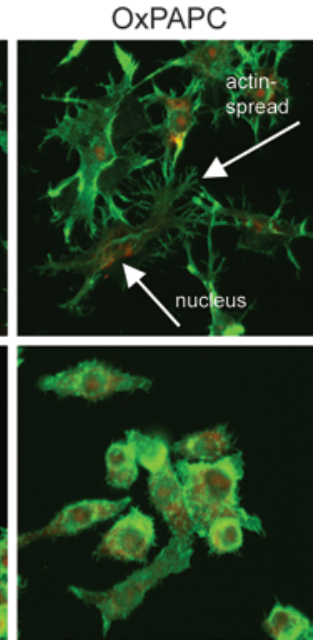

\section{Figure 1}

Oxidation of lipids occurs in E. coli peritonitis in vivo and leads to an actin-dependent change in cell shape in vitro. (A) Endogenous levels of oxidized phosphatidylcholine were measured in PLF of mice infected with E. coli after 8 or 16 hours, respectively, compared with supernatants of RAW 264.7 cells after adding 5 or $10 \mu \mathrm{g} / \mathrm{ml}$ of OxPAPC, respectively. Co, control. (B) RAW 267.4 cells were incubated with indicated doses of OxPAPC or DMPC for 15 minutes, and phagocytosis of E. coli was assessed after 60 and 120 minutes (triplicates, representative of 3 independent experiments). (C) FACS histogram showing uptake of FITC-labeled E. coli by resident peritoneal macrophages pretreated with $10 \mu \mathrm{g} / \mathrm{ml}$ of OxPAPC or DMPC after 60 minutes. (D) Mice ( $n=8 /$ group) were infected with $10^{4}$ CFU E. coli i.p. and treated with $2.5 \mathrm{mg} / \mathrm{kg}$ DMPC or OxPAPC i.p. Peritoneal CFU counts were enumerated 10 hours after infection. Data (A-D) are presented as mean \pm SEM; ${ }^{*} P<0.05 ;{ }^{* *} P<0.01$ versus controls. ${ }^{* \star *} P<0.001$. (E) RAW 264.7 cells were incubated with carrier, DMPC, or OxPAPC $(10 \mu \mathrm{g} / \mathrm{ml} ; 30$ minutes $)$ alone or following incubation with $2 \mu \mathrm{M}$ cytochalasin D (30 minutes). Cells were subsequently stained for F-actin using phalloidin (green) and PI for nuclei (red). Scale bar: $30 \mu \mathrm{m}$.

measured with a monoclonal antibody that recognizes the phosphocholine headgroup of OxPL (Figure 1A and ref. 21). Quantification of OxPL generated during E. coli peritonitis in vivo as compared with OxPL levels in cell supernatants following exogenous administration of OxPL in vitro demonstrated comparable amounts (Figure 1A). More detailed analyses demonstrated that these equivalent amounts of OxPL (Figure 1A) reduced the uptake of bacteria by peritoneal macrophages in a dose-dependent manner (Figure 1, B and C). Consequently, administration of OxPL led to enhanced bacterial loads in the peritoneal cavity (Figure 1D). Control experiments confirmed that delivery of native phospholipids did not have this effect (Figure 1D).

The changes in cell shape associated with phagocytosis require the active remodeling of actin (22). Delivery of OxPL also affects actin polymerization (23). Further support for this notion was provided by fluorescent imaging of RAW 264.7 macrophages showing that treatment with OxPL induced cell spreading, which is a hallmark of actin reorganization (Figure 1E). This phenomenon was not observed in control experiments in which RAW
264.7 macrophages were treated with unoxidized phospholipids or cytochalasin $\mathrm{D}$, a chemical inhibitor of actin polymerization (Figure 1E). Quantification of cell perimeter and area using CellProfiler cell image analysis software confirmed that OxPL treatment induced spreading of cells (Supplemental Figure 1; supplemental material available online with this article; doi:10.1172/ JCI60681DS1). Related studies have suggested that this proceeds through a pathway where CD36 is a receptor for OxPL $(24,25)$. More detailed analyses attempted to assess the contribution of the CD36 scavenger receptor in mediating these OxPL effects. Interestingly, we could not discern a role for CD36 in OxPL-mediated cell spreading when experiments were performed in CD36 mutant oblivious (CD36 ${ }^{\mathrm{obl}}$ ) peritoneal macrophages, which harbor a nonfunctional scavenger receptor (Supplemental Figure 2A). Further analyses in these cells demonstrated that although the absence of functional CD36 affected phagocytosis of E. coli when compared with WT macrophages, this process is still suppressed in the presence of OxPL (Supplemental Figure 2B). A number of other receptors have been described to be important in OxPL- 
A
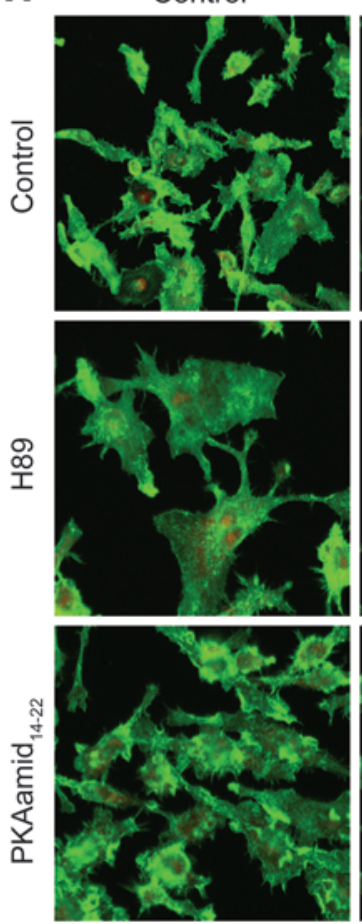

$\mathbf{F}$
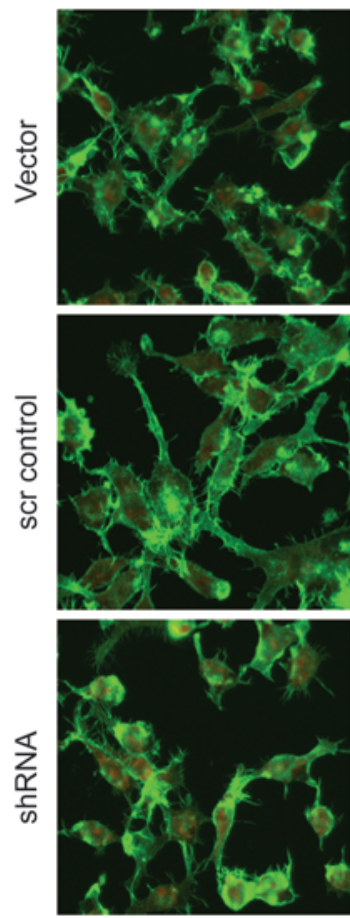

DMPC
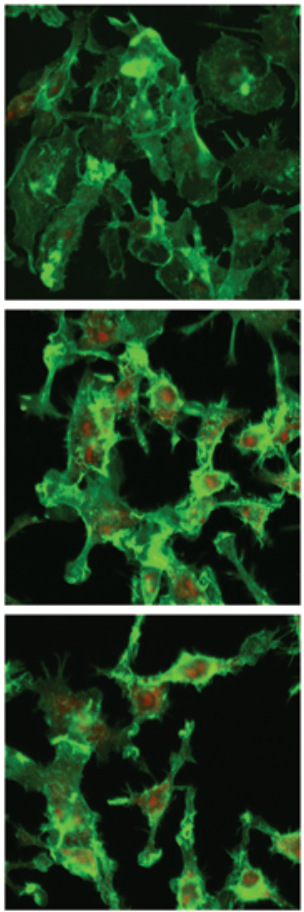

DMPC
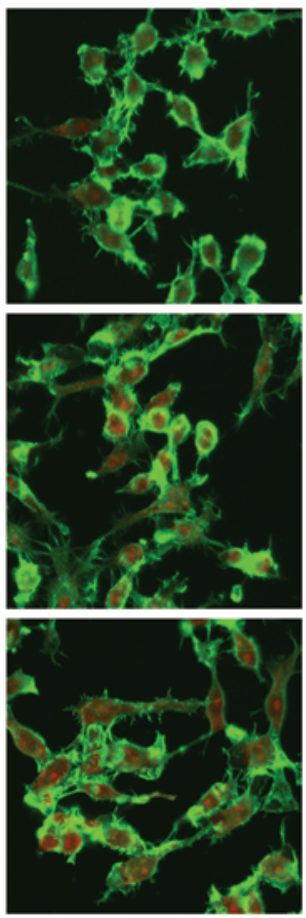

OxPAPC
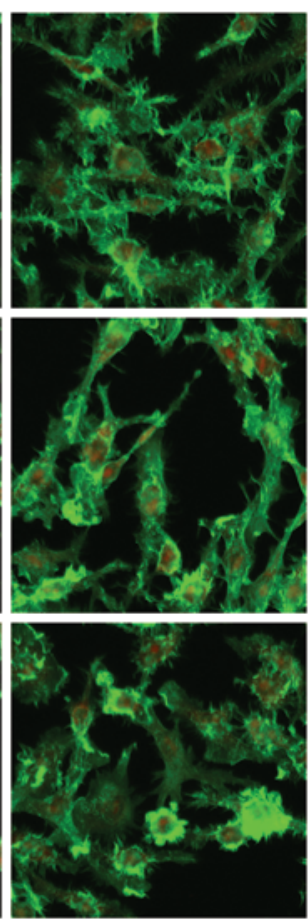

OxPAPC
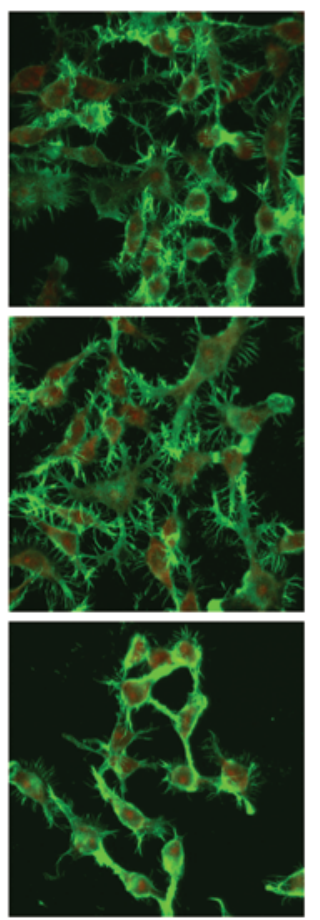
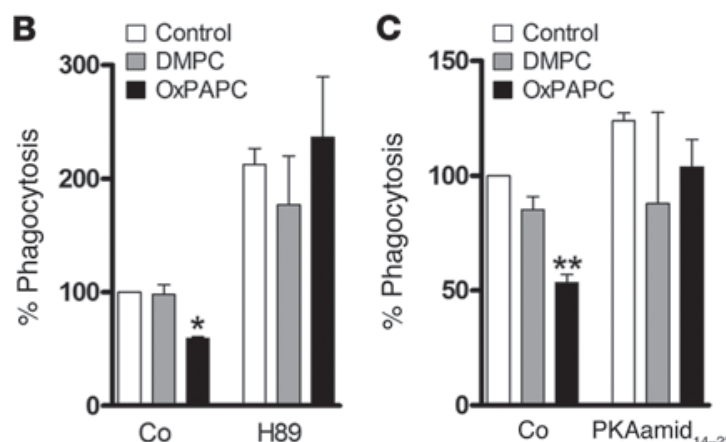

D
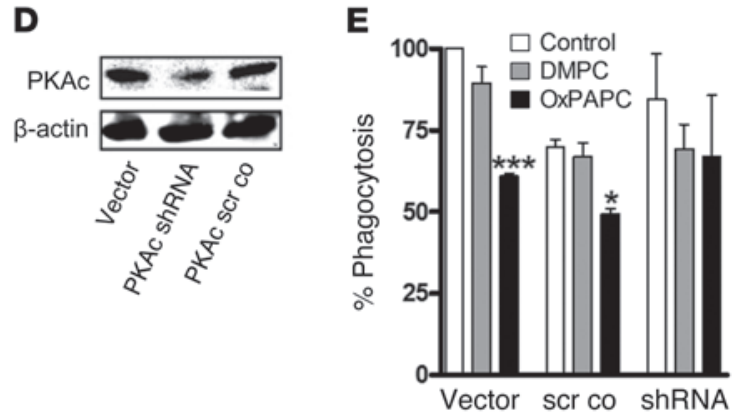

G

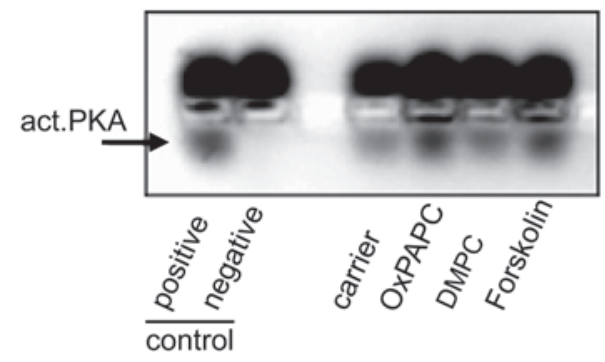

Figure 2

PKA activation mediates OxPAPC-associated cell spread and inhibition of phagocytosis. (A-C) RAW 264.7 cells were treated with carrier, DMPC, or OxPAPC $(10 \mu \mathrm{g} / \mathrm{ml}, 30$ minutes in A; $5 \mu \mathrm{g} / \mathrm{m}, 15$ minutes in $\mathbf{B}$ and $\mathbf{C}$ ) alone or following preincubation with H89 $(10 \mu \mathrm{M})$ or PKA amide ${ }_{14-22}(20 \mu \mathrm{M})$ (30 minutes). (A) Cells were subsequently stained for F-actin (phalloidin; green) and PI (red). (B and C) Uptake of FITC-labeled E. coli was evaluated after 60 minutes and is expressed relative to carrier. (D) RAW 264.7 cells were transfected with shRNA to the $\alpha$-isoform of PKAc, and silencing was verified by Western blot. ( $E$ and $\mathbf{F}$ ) Control (vector or scrambled control) and shRNA-transfected cells were preincubated with carrier, DMPC, or OxPAPC $(5 \mu \mathrm{g} / \mathrm{ml}$, 15 minutes in $\mathbf{E} ; 10 \mu \mathrm{g} / \mathrm{ml}, 30$ minutes in F). (E) Phagocytosis of FITC-labeled E. coli was examined after 60 minutes and is expressed relative to carrier. (F) Cells were stained with phalloidin-Alexa Fluor 488 (green) and PI (red). Representative images of 3 independent experiments are shown. (G) RAW 264.7 cells were incubated with DMPC or OxPAPC at $10 \mu \mathrm{g} / \mathrm{ml}$, forskolin $(100 \mu \mathrm{M})$, or carrier for 15 minutes. PKA activity was measured as described in Methods. Arrow indicates activated PKA (lower band); "positive" and "negative" indicate assay control. Data are mean \pm SEM of triplicates and representative of 3 independent experiments; ${ }^{*} P<0.05 ;{ }^{* *} P<0.01$; ${ }^{* *} P<0.001$ versus corresponding carrier. Original magnification, $\times 800$. 
A
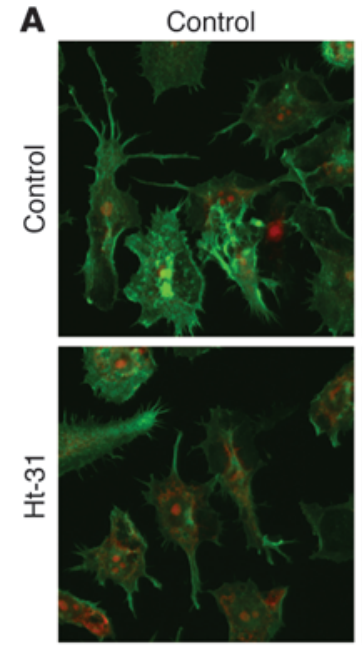

c

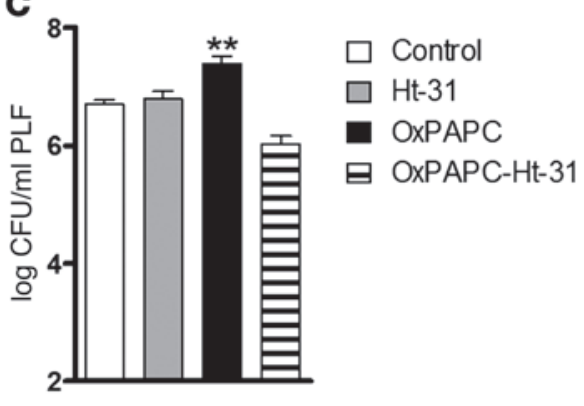

DMPC
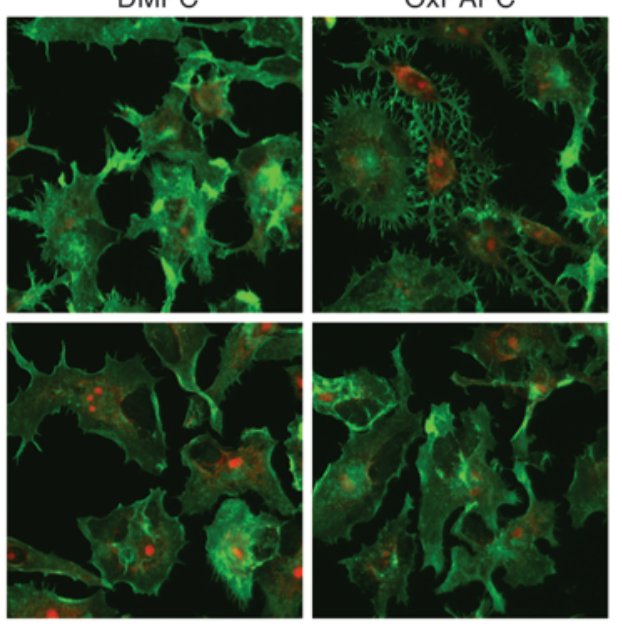

D

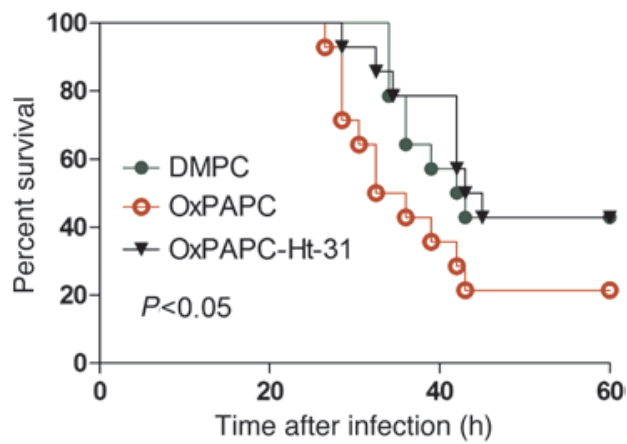

Figure 3

OxPL-induced inhibition of phagocytosis requires anchoring of PKA in vivo and in vitro. (A) RAW 264.7 cells were incubated with carrier, DMPC, or OxPAPC $(10 \mu \mathrm{g} / \mathrm{ml})$ alone or after treatment with $100 \mu \mathrm{M} \mathrm{Ht}-31$ (30 minutes) and stained with phalloidin (green) and PI (red). Original magnification, $\times 800$. (B) RAW 264.7 cells were treated with carrier or phospholipids $(5 \mu \mathrm{g} / \mathrm{ml} ; 15$ minutes) alone or after preincubation with Ht-31 $(100 \mu \mathrm{M})$ for 30 minutes. Phagocytosis of FITC-labeled E. coli was analyzed using FACS after 60 minutes. Uptake is expressed relative to carrier. ${ }^{\star \star} P<0$.01. Data show mean \pm SEM of triplicates and are representative of 3 independent experiments. (C) Mice received carrier or $2.5 \mathrm{mg} / \mathrm{kg}$ OxPAPC i.p. and/or $100 \mu \mathrm{M}$ of Ht-31 immediately before infection with $10^{4} \mathrm{CFU}$ E. coli. At 10 hours after infection, PLF was harvested and bacterial CFUs enumerated. Data are mean \pm SEM of 2 independent experiments from $n=7-9$ mice/group; ${ }^{\star *} P<0.01$ versus carrier. (D) Mice received $2.5 \mathrm{mg} / \mathrm{kg}$ DMPC or OxPAPC i.p. followed by i.p. injection of vehicle or Ht-31 (OxPAPC-Ht-31), after which they were infected with $10^{4} \mathrm{CFU}$ E. coli. Survival was monitored every 2 hours; $n=12$ mice/group. $P$ values indicate differences between OxPAPC versus DMPC or OxPAPC versus OxPAPC-Ht-31, respectively.

mediated effects, such as the platelet-activating factor receptor (PAFR) and the prostaglandin E2 (EP2) receptor $(26,27)$. However, chemical inhibition of these receptors was neither able to abrogate OxPL-mediated spreading nor the inhibition of phagocytosis, suggesting that in our cell type and system, neither CD36 (Supplemental Figure 2) nor PAFR or EP2 receptor plays any role (data not shown). To exclude the possibility of toxic effects exerted by OxPL, we performed phagocytosis experiments in which phospholipids were removed after preincubation, but prior to addition of bacteria. These experiments showed that the inhibitory effects of OxPL were fully reversible over time (Supplemental Figure 3). Consistent with previous observations, this reversal was slow, suggesting that OxPL could affect cell-associated factors, including lipid raft or caveolae organization as described (28-30). Collectively, our data argue against toxic effects or competition for receptors shared between E. coli and OxPL. Rather, they imply that downstream signaling events account for OxPL-mediated effects on phagocytosis and cell spreading.
Cell spreading via specific PKA activation by OxPL inhibits phagocytosis. Next, we focused on assessing the relative contributions of phosphoinositide and cAMP-dependent signaling pathways in the control of OxPL-mediated phagocytosis and cell spreading. Activation of PI3K and the concomitant mobilization of Rho family small GTPases have been implicated in actin-remodeling events in macrophages $(17,23,31)$. However, treatment with pharmacological agents that target PI3K (wortmannin at $50 \mathrm{nM}$ and LY294002 at $10 \mu \mathrm{M}$ ) and Rho GTPases (Rho kinase inhibitor Y27632 at 10 $\mu \mathrm{M}$ and clostridium toxin B at $100 \mathrm{ng} / \mathrm{ml}$, which inactivates Rho, $\mathrm{Rac}$, and $\mathrm{Cdc42}$ ) had no effect on OxPL action in macrophages (Supplemental Figure 4 and data not shown). Mobilization of cAMP-responsive events is known to suppress receptor-mediated phagocytosis in macrophages (32). Accordingly, pretreatment with pharmacological inhibitors of PKA, including H89 and PKA amide $_{14-22}(33,34)$, abolished OxPL-induced actin spread (Figure 2A) and completely abrogated the inhibition of phagocytosis (Figure 2, B and C). However, H89 did increase baseline phagocytosis 
A

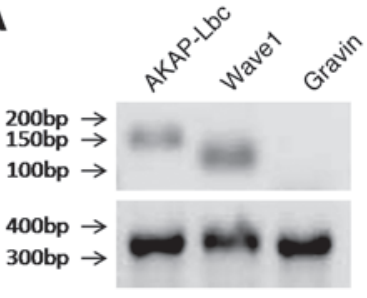

D
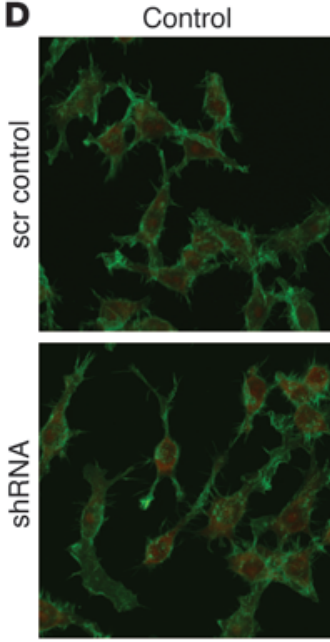

I
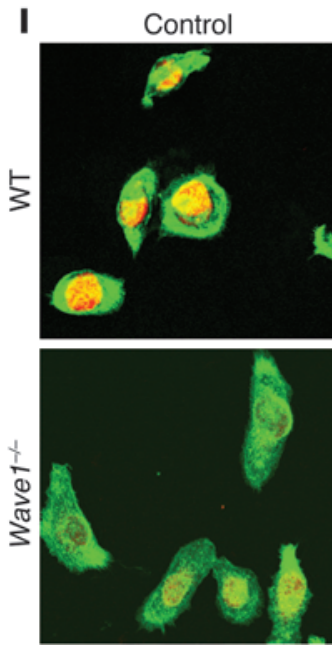

B

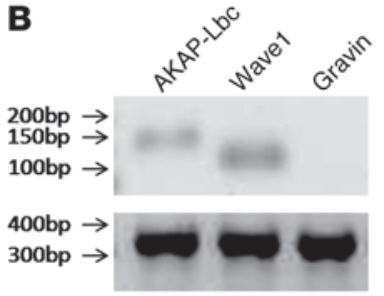

DMPC
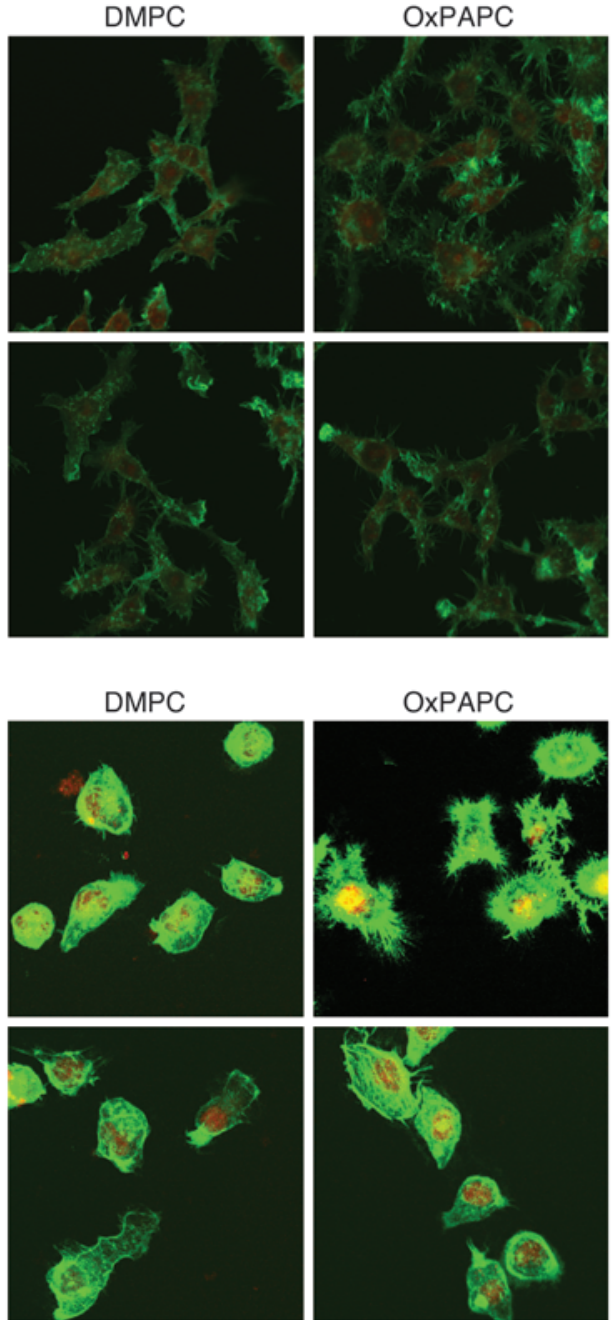

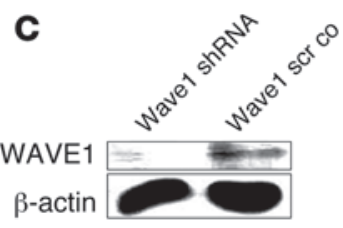

E
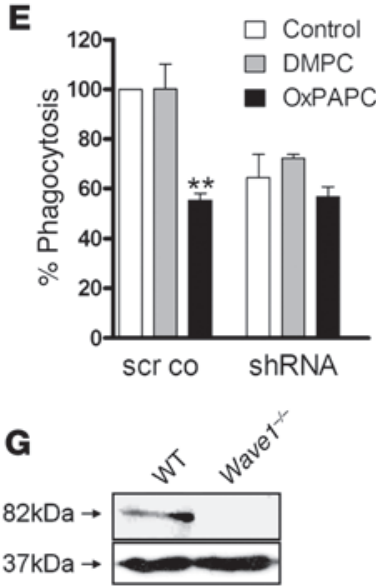

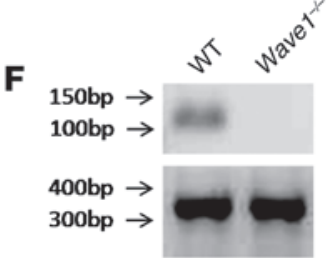

H

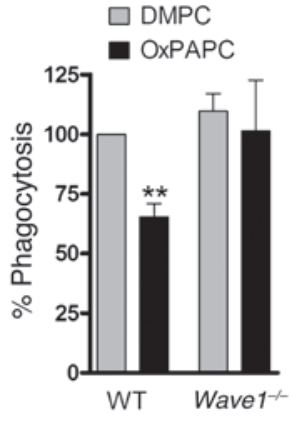

Figure 4

The AKAP WAVE1 mediates OxPL inhibition of phagocytosis in peritoneal macrophages. AKAP-Lbc (150 bp), Wave1 (116 bp), and Gravin (136 bp) mRNA expression in (A) RAW 264.7 or (B) primary peritoneal macrophages; GAPDH (372 bp). (C) Western blot verifying silencing of Wave1 (82 kD) in RAW 264.7 cells; $\beta$-actin control (37 kD). (D) Scrambled control and shRNA (targeting Wave1) cells incubated with carrier, DMPC, or OxPAPC $(10 \mu \mathrm{g} / \mathrm{ml})$ and stained with phalloidin (green) and PI for nuclei (red). Original magnification, $\times 800$. (E) Phagocytosis of $E$. coli (60 minutes) assayed in scrambled control and shRNA-Wave 1 cells preincubated with carrier, DMPC, or OxPAPC $(5 \mu \mathrm{g} / \mathrm{ml})$. Data depicted are mean \pm SEM of triplicates, ${ }^{*} P<0.05$ versus corresponding carrier/DMPC. (F) mRNA expression and (G) Western blot for Wave1 in WT and Wave $1^{-/-}$primary peritoneal macrophages (WAVE1 $82 \mathrm{kD} ; \beta$-actin $39 \mathrm{kD}$ ). (H) Primary peritoneal macrophages of WT and Wave $1^{-/}$mice incubated with carrier, DMPC, or OxPAPC $(10 \mu \mathrm{g} / \mathrm{ml})$ and stained with phalloidin (green) and PI (red). (I) Phagocytosis of FITC-labeled E. coli (60 minutes) by WT and Wave $1^{-1-}$ peritoneal macrophages analyzed after prior incubation with DMPC or OxPAPC $(5 \mu \mathrm{g} / \mathrm{ml})$. Data are mean \pm SEM of triplicates of 2 independent experiments; ${ }^{* *} P<0.01$ versus corresponding DMPC.

of the dimyristoyl-phosphatodyl-choline (DMPC) control. This nonspecific effect was lower for PKA amide ${ }_{14-22}$, a more specific inhibitor of PKA than H89, which binds to the catalytic subunit of PKA in the nanomolar range (refs. 35-37 and Figure 2, B versus
C). More definitive results were obtained with gene silencing of the $\alpha$-isoform of the catalytic subunit of PKA using shRNA, which successfully reversed OxPL-associated inhibition of phagocytosis and reduced OxPL-induced spreading (Figure 2, D-F). These find- 

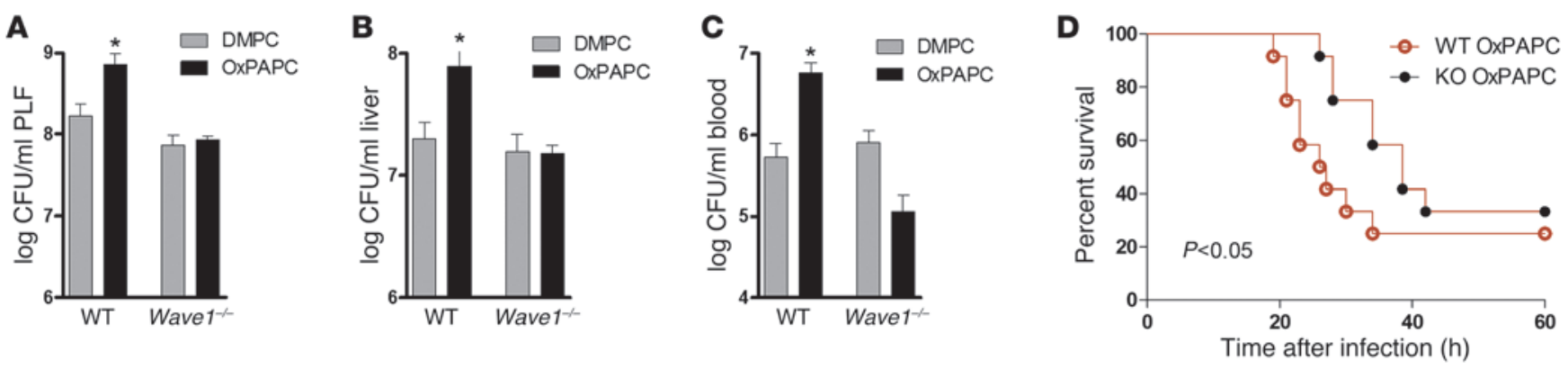

Figure 5

Chimeric Wave1-KO mice are rendered unresponsive to OXPL. (A-D) WT mice reconstituted with WT or Wave1 ${ }^{-/-}$bone marrow were treated with DMPC or OxPAPC (2.5 mg/kg) i.p. and infected with 104-5 CFU E. coli i.p. (A) Peritoneal, (B) liver, and (C) blood CFU counts were enumerated 10 hours after infection, and (D) survival was monitored every 2 hours. Data are from $n=9-12$ mice/group and are presented as mean \pm SEM; ${ }^{*} P<0.05$ versus corresponding DMPC control.

ings led us to hypothesize that OxPL itself activates PKA. Indeed, incubation with OxPL but not native phospholipids (DMPC) increased PKA activity (Figure 2G). Of note, we observed that forskolin that activates PKA via cAMP was not able to mimic the antiphagocytic effects of OxPL (Supplemental Figure 5), strongly suggesting that although PKA's catalytic activity was necessary for OxPL-mediated effects and that OxPL can induce PKA phosphorylation, the localization of PKA is essential for OxPL-mediated effects. Taken together, these results allow us to demonstrate that OxPL activates PKA, which in turn propagates cellular events associated with actin spread and inhibition of phagocytosis.

AKAP inbibition prevents detrimental effects of OxPAPC in vitro and in vivo. It is widely acknowledged that PKA phosphorylation events control a plethora of processes and that the specificity of this kinase in different cellular compartments is directed through interaction with AKAPs $(38,39)$. To investigate whether PKAAKAP interaction is required for OxPL-induced effects, we exploited a cell-permeable AKAP-inhibitory peptide (stearated Ht-31) that blocks association of the regulatory subunit RII of PKA with AKAPs (40). Preincubating macrophages with Ht-31 abrogated the change in cell shape caused by OxPL (Figure 3A) and concomitantly prevented OxPL-associated inhibition of phagocytosis (Figure 3B). Notably, administration of Ht-31 together with OxPL at the onset of E. coli peritonitis in mice prevented the increase in bacterial loads caused by OxPL in vivo (Figure 3C). Survival analysis corroborated these findings, as disruption of PKA anchoring with $\mathrm{Ht}-31$ peptide was able to reverse the detrimental effects of OxPL during E. coli peritonitis in vivo (Figure 3D). These data strongly suggest that AKAP interactions contribute to OxPL-induced PKA activation, resulting in diminished phagocytosis of bacteria.

WAVE1 mediates antiphagocytic properties of OXPL in vitro. Among the 50 AKAPs discovered thus far, only Gravin, AKAP-Lbc, and WAVE1 are thought to interface with the actin cytoskeleton (38). However, only AKAP-Lbc and WAVE1 are expressed in RAW 264.7 cells and primary peritoneal macrophages (Figure 4, A and B, and Supplemental Figure 6). shRNA-mediated gene-silencing techniques revealed that knockdown of WAVE1 suppressed OxPLassociated actin spread and inhibition of phagocytosis (Figure 4, C-E). In contrast, gene silencing of AKAP-Lbc had no effect (data not shown). Given that WAVE1 shRNA also resulted in a decrease in baseline phagocytosis (Figure 4E), more definitive support for this concept was provided when similar experiments were performed in primary peritoneal macrophages isolated from Wave1 $1^{-/-}$ mice (Figure 4, F-H, and ref. 41). Wave 1/- $^{-1}$ acrophages exhibited neither cell spreading nor impaired bacterial uptake upon OxPL treatment (Figure 4, H and I). These results imply that a pool of PKA associated with WAVE1 modulates cell spreading and inhibition of phagocytosis in macrophages.

Knock out of Wave1 prevents detrimental effects of OxPL in E. coli peritonitis. We then evaluated the role of WAVE1 during E. coli peritonitis in vivo. To exclude the potential influence of the altered size of Wave $1^{-/-}$mice (41), we generated chimeric mice on a C57BL/6 background. For this we administered bone marrow of Wave $1^{-/-}$or WT littermates to lethally irradiated C57BL/6 mice and ensured complete reconstitution with Wave1 ${ }^{-1-}$ donor peritoneal macrophages after 9 weeks (Supplemental Figure 7 and ref. 42). Following i.p. injection of either DMPC or OxPL, we infected mice with E. coli i.p. and examined their ability to contain bacterial dissemination. OxPL treatment led to enhanced bacterial outgrowth in mice that received WT bone marrow (Figure 5, A-C). In contrast, chimeric mice with Wave $1^{-/}$peritoneal macrophages appeared resistant to the effects of OxPL (Figure 5, A-C). The CFU count in PLF, liver, and blood was similar to the values measured in control mice that received unoxidized lipids. Moreover, Wave $1^{-/-}$macrophages seemed resistant to the OxPL-associated impairment of survival during E. coli peritonitis (Figure 5D). Collectively, these data confirm that WAVE1 mediates the inhibition of phagocytosis caused by OxPL in vitro and in vivo.

Endogenously generated OxPL inhibit phagocytosis in a WAVE1-dependent manner. To finally study the potential clinical implication, we investigated the direct impact of endogenously generated OxPL on bacterial phagocytosis. We searched for conditions of chronic inflammation with access to body fluids. We chose to collect peritoneal dialysis fluid (PDF) from patients with renal failure, as we anticipated this to contain enhanced levels of OxPL due to impaired antioxidant defense mechanisms, increased oxidative stress (43-45), and the risk for recurrent bacterial peritonitis and sepsis $(46,47)$, symptomatic for this group of patients. Therefore we studied PDF for the presence and activity of OxPL. OxPL could be detected in PDF from peritoneal dialysis patients (Figure 6A). Incubation of primary murine peritoneal macrophages with human PDF per se did not influence bacterial phagocytosis. However, upon removal of antibodies via protein $\mathrm{G}$ beads or heat inactivation (data not shown), human PDF particularly inhibited phagocytosis (Figure 6B and Supplemental Figure 8A), suggesting that contaminating proteins and antibodies compensated for the 
A

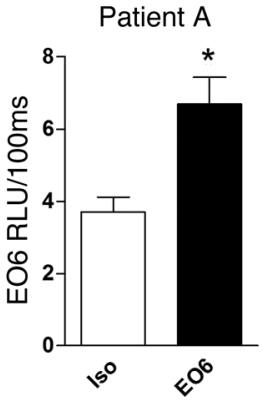

D

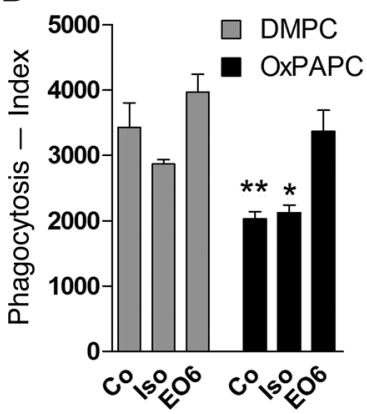

Patient B

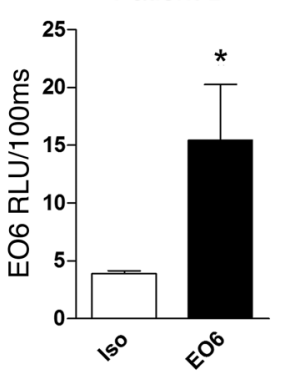

B

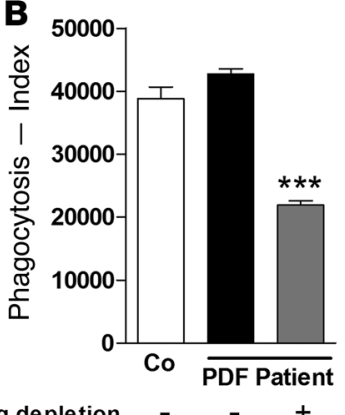

E Chloroform extraction:

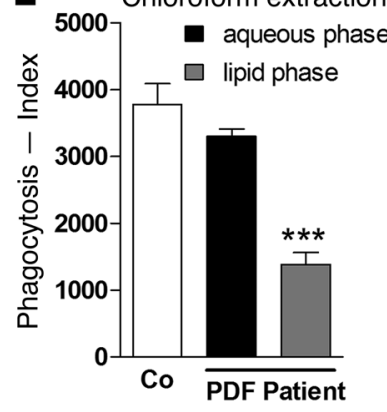

$\mathbf{F}$

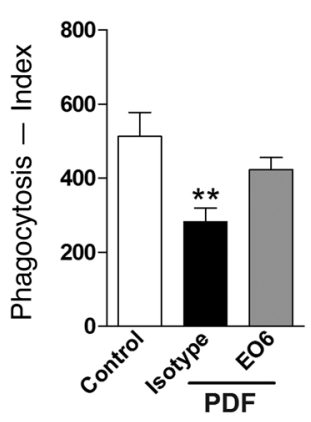

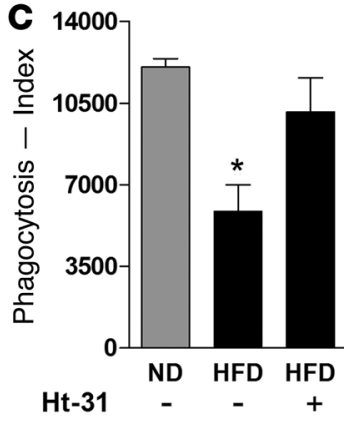

G

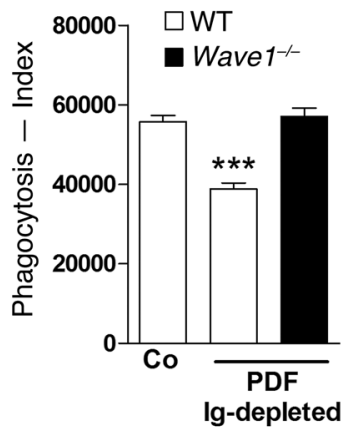

Figure 6

OxPL in human PDF inhibit phagocytosis in the presence of WAVE1. (A) Levels of oxidized phosphocholine were measured using the EO6 mAb or an isotype control in PDF from 2 patients undergoing peritoneal dialysis. (B) Murine primary peritoneal macrophages were incubated for 15 minutes with PDF of 1 representative patient (patient A), and phagocytosis of E. coli after 60 minutes was assessed by FACS. Ig depletion was done with protein G beads. (C) PLF from Ld/r-1-Rag-/- mice on normal diet (ND) or high-fat diet (HFD) was placed on primary peritoneal macrophages in the presence or absence of $\mathrm{Ht}-31(100 \mu \mathrm{M})$ and phagocytosis of E. coli after 60 minutes. (D) RAW 264.7 macrophages were either preincubated with EO6 antibody or isotype control $(1 \mu \mathrm{g} / \mathrm{ml})$ for 1 hour, then with OxPL or DMPC $(5 \mu \mathrm{g} / \mathrm{ml})$ for 15 minutes. Phagocytosis of E. coli was assessed after 60 minutes by FACS. Control assays were done in RPMI. (E) PDF of patient A was subjected to chloroform extraction, and the resultant water or lipid fraction was added to RAW 264.7 cells 15 minutes prior to addition of E. coli. Phagocytosis was assayed after 60 minutes by FACS. Control assays were done in RPMI. (F) RAW 264.7 macrophages were incubated for 15 minutes with IgG-depleted PDF that had been pretreated with either EO6 or isotype control antibody $(10 \mu \mathrm{g} / \mathrm{ml})$ for 1 hour, and phagocytosis of E. coli after 60 minutes was assessed by FACS. (G) WT and Wave 1-/ primary peritoneal macrophages were incubated with Ig-depleted PDF from patient A, and phagocytosis of E. coli was determined was analyzed. Data are mean \pm SEM of at least duplicate experiments; ${ }^{*} P<0.05 ;{ }^{* \star} P<0.01 ;{ }^{* \star *} P<0.001$ versus corresponding control.

inhibitory effects of OxPL. Further confirmation that antibodies masked the antiphagocytic effects of OxPL was obtained by showing that PLF from Ldlr/Rag-DKO mice that contained elevated OxPL levels upon a high-fat diet (data not shown) but lacked antibodies due to the absence of $\mathrm{B}$ cells inhibited bacterial phagocytosis (Figure 6C). This inhibitory effect of PLF was abolished in the presence of H89 (not shown) or Ht-31 (Figure 6C). Ldlr/ Rag-DKO mice not only lack IgG but also IgM. EO6 is a prototypic natural IgM antibody that specifically recognizes the phosphocholine head group of OxPL and was shown earlier to neutralize OxPL-mediated effects $(21,48,49)$. We show here that EO6 was also able to reverse the OxPL-mediated inhibition of phagocytosis (Figure 6D). Strengthening the biological role of lipids such as OxPL in human PDF, we discovered that only the lipid phase of chloroform-extracted PDF potently inhibited phagocytosis (Figure 6E), whereas lipid removal from Ig-depleted PDF with charcoal reversed the inhibitory effect (data not shown). In line with Figure $6 \mathrm{D}$, neutralization of OxPL by treatment with EO6 antibodies reversed the inhibitory effect on phagocytosis by Ig-depleted human PDF (Figure 6F). Last but not least, inhibition of phagocytosis by Ig-depleted human PDF was reversed in the absence of
WAVE1 (Figure 6G and Supplemental Figure 8B). In concert, these data illustrate that WAVE1 mediates inhibition of phagocytosis by endogenously generated OxPL that can be detected in clinical specimens such as PDF.

\section{Discussion}

In this study, we investigated the mechanism by which OxPL affect phagocytosis during E. coli peritonitis. We have discovered what we believe is a previously unrecognized role of the cytoskeletalassociated AKAP WAVE1 in macrophages.

WAVE1 belongs to the WASP family, which controls actin polymerization via the Arp2/3 complex (18). In contrast with other WASP family members, WAVE1 also functions to anchor PKA and the Abl tyrosine kinase at sites of actin reorganization (19). So far, the majority of WAVE1 action has been studied in the brain. Wave1 $1^{-/-}$ mice exhibit altered synaptic transmission, depleted neuronal migration, behavioral deficits, and reduced viability $(41,50)$. These electrophysiological and behavioral deficits have been traced back to abnormalities in dynamic actin polymerization and dendritic spine morphology (51). We have now uncovered an unanticipated role for WAVE1 and PKA in innate immunity. Three lines of evidence 
support this claim: (a) inhibition of PKA and disruption of PKA anchoring suppress OxPL-induced cell spreading and phagocytosis (Figures 2 and 3); (b) WAVE1 was recently found to be expressed in bone marrow-derived macrophages (20); and (c) gene silencing of WAVE1 or ablation of this AKAP gene in peritoneal macrophages protect against OxPL challenge in situ and in vivo (Figures 4-6).

We were able to corroborate previously described findings by making use of biological samples that contained endogenously enhanced levels of OxPL. Elevated levels of OxPL present in PDF from patients with renal failure undergoing peritoneal dialysis or in PLF from $L d l r / R a g-D K O$ mice on a high-fat diet diminished bacterial phagocytosis. In either case, disruption of PKA anchorage by administration of Ht-31 or WAVE1 deficiency proved sufficient to restore phagocytosis (Figure 6 and Supplemental Figure 8).

Several clinical conditions, such as end-stage renal failure, cardiovascular disease, and type 2 diabetes, are characterized by chronic inflammation and an increased risk for severe bacterial infection $(47,52-54)$. The reason for this enhanced susceptibility to bacterial infections is not well understood (53). Recent reports emphasized the tremendously enhanced risk of death from sepsis and infections in patients with renal failure, with mortality from sepsis being 50 times higher in patients on dialysis as compared with the general population $(47,55)$. We hypothesized that in these patients, chronic inflammation and the resulting generation of oxidation products such as OxPL might importantly contribute to impaired host defense by inhibiting phagocytosis of bacteria. To address this idea we selected end-stage renal failure patients undergoing peritoneal dialysis, since oxidative modifications in PDF and altered phagocytosis in the peritoneal compartment were observed earlier (44, $45,56,57)$. Our investigations clearly enabled us to demonstrate a role for OxPL in inhibiting phagocytosis in these patients (Figure 6) and furthermore allowed us to demonstrate the importance of the AKAP WAVE1 herein. Of note, the presence of endogenous antibodies and possibly other serum components partly masked the effects of endogenous OxPL in PDF. The importance of antibodies and complement in facilitating phagocytosis is well established (17) and explains that IgG-depleted PDF (Figure 6, B and E) or PLF from antibody-deficient $L d l r / R a g$-DKO (Figure 6C) mice unleashed the pronounced effect of OxPL on phagocytosis. In line with this, higher incidences of bacterial peritonitis were reported in children on peritoneal dialysis with reduced antibody levels (58).

While IgG directly augments phagocytosis via opsonization of bacteria, serum components such as C-reactive protein and natural antibodies are able to bind and specifically neutralize the biological effects of OxPL $(37,38)$. We confirm the neutralizing role of natural antibodies, since the natural IgM antibody EO6 that recognizes and blocks the phosphocholine residue of OxPL was sufficient to abolish the antiphagocytic effects of OxPL (Figure 6D) as well as Ig-depleted human PDF (Figure 6F). These data not only reveal that EO6 prevents the antiphagocytic effects of OxPL but furthermore provide some evidence as to the active component in oxidized 1-palmitoyl-2-arachidonoyl-sn-glycero-3-phosphorylcholine (OxPAPC), since the biological activities of POVPC, PEIPC, and PECPC, but not PGPC, have been shown earlier to be particularly neutralized by this antibody $(59,60)$.

During acute infections, host defense mechanisms are primarily aimed at establishing a highly phagocytic milieu, which might be the reason why we did not observe a more pronounced, although still significant, effect of OxPL in this murine sepsis model. Given the protective mechanisms outlined above, we deem it still remarkable that WAVE1 deficiency was sufficient to protect against the detrimental effects of these DAMPs. We also performed cecal ligation and puncture (CLP) experiments, but could not observe any effect of OxPL and therefore also not WAVE1 in this model (data not shown). This indirectly confirms that OxPL predominantly affect phagocytosis because phagocytosis of bacteria is not that essential after CLP due to abscess formations around the cecum with containment of bacteria (61). As such, it is known that the lack of antibodies is not associated with an altered outcome from CLP (62), whereas Rag-deficient mice with $E$. coli peritonitis suffer from impaired bacterial clearance and worsened survival (63).

Collectively, these findings not only underscore the advantages of PKA anchoring as a means to enhance the selectivity of cAMPresponsive events, but also unearth an additional role for the WAVE1 signaling complex. We are the first, to our knowledge, to describe the function of WAVE1 in macrophages and a role for WAVE1 in innate immunity. However, it is important to note that WAVE1 affected phagocytosis only in the presence of OxPL, thus indicating a requirement for oxidative stress as seen during serious inflammatory diseases or infections. Although the precise contribution of OxPL in the E. coli peritonitis model and in patients with renal failure discussed in this report is not yet fully understood, an important implication of this study is that diminished bacterial clearance is attenuated by interfering with a WAVE1-associated pool of PKA.

The lack of effective therapies to combat sepsis (64) and the enhanced risk for bacterial infections in patients with chronic inflammatory diseases makes it tempting to speculate that targeting OxPL's negative impact on bacterial phagocytosis by WAVE1 inhibitors might prove to be a promising future direction for therapeutic intervention.

\section{Methods}

Phospholipids. PAPC and DMPC were obtained from Sigma-Aldrich. DMPC was used as control lipid, as it lacks unsaturated fatty acids and thus cannot be oxidized. OxPAPC was generated by air oxidation (11) and the extent of oxidation confirmed by electrospray ionization-mass spectrometry (42). Only preparations showing a reproducible pattern of lipid oxidation products were used, and after testing for biological activity and exclusion of LPS contamination using the Limulus assay.

Phagocytosis assays. Phagocytosis assays were performed as described previously (12). Briefly, RAW 264.7 cells or primary resident peritoneal macrophages were plated at $0.5 \times 10^{6} / \mathrm{ml}$ in 12 -well microtiter plates (Greiner) and allowed to adhere overnight. After washing steps, RPMI was added to wells, and macrophages were incubated for 15 minutes with OxPAPC, DMPC $(5 \mu \mathrm{g} / \mathrm{ml}$, unless otherwise indicated), or saline (control). In selected experiments, cells were preincubated for 15 minutes with PDF from patients. Subsequently, FITC-labeled heat-killed E. coli (O18:K1) at a MOI of 100 was added for 1 hour at $37^{\circ} \mathrm{C}$ or $4^{\circ} \mathrm{C}$, respectively. To remove adherent but not internalized bacteria, cells were treated with proteinase $\mathrm{K}$ at $50 \mu \mathrm{g} / \mathrm{ml}$ for 15 minutes at room temperature. Immediately thereafter, cells were placed on ice, washed, and analyzed using a FACScan (BD). The phagocytosis index of each sample was calculated as follows: (mean fluorescence $\times$ percentage of positive cells at $37^{\circ} \mathrm{C}$ ) minus (mean fluorescence $\times$ percentage of positive cells at $4^{\circ} \mathrm{C}$ ). Pretreatment with pharmacological inhibitors was performed as indicated.

Mice. Pathogen-free C57BL/6 mice were purchased from Charles River. $\mathrm{CD} 36^{\text {obl }} \mathrm{C} 57 \mathrm{BL} / 6$ mice were provided by Bruce Beutler via the Mutant Mouse Regional Resource Centers (MMRC) (65). Wave1 $1^{-/-}$mice were gener- 
ated as described (41) and backcrossed 10 times to a C57BL/ 6 background. $\mathrm{Ldlr}^{-/}$were crossed with $\mathrm{Rag}^{-/-}$mice (Jackson), and backcrossed 10 times to a C57BL/6 background to generate $\mathrm{Ldlr}^{/ /} \mathrm{Rag}^{/ /}$mice. Male $\mathrm{Ldlr}^{/ /} \mathrm{Rag}^{/-}$ mice housed under specific pathogen-free conditions were fed on a regular chow diet for 8-10 weeks and then switched to an atherogenic diet containing $21 \%$ fat and $0.2 \%$ cholesterol (TD88137; Ssniff Spezialdiäten GmbH) for an additional 8-10 weeks.

Induction of peritonitis, enumeration of bacteria, and monitoring of survival. Peritonitis was induced by i.p. injection of $200 \mu \mathrm{l}$ saline containing $10^{4}$ to $10^{5}$ CFUs E. coli 018:K1 that were harvested at mid-log phase (12). OxPAPC or DMPC (both at $2.5 \mathrm{mg} / \mathrm{kg}$ ) were administered i.p. imme-

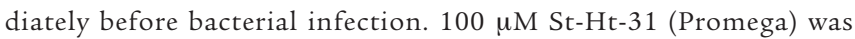
injected i.p. immediately before administering lipids and bacteria. In survival studies, 12 mice/group were inoculated with E. coli, and mortality was assessed every 2 hours. For quantification of bacteria, PLF and organs were harvested 10 hours after infection and processed for bacterial quantification as described (12).

Measurement of oxidized lipids. To prevent oxidation, all samples (human and mouse) were supplemented with butylated hydroxytoluene $(0.01 \%)$ and diethylene triamine pentaacetic acid (DTPA) immediately after collection, purged with nitrogen, and stored in aliquots at $-70^{\circ} \mathrm{C}$. PLF and PDF samples were adjusted to $100 \mu \mathrm{g} / \mathrm{ml}$ protein concentration in PBS containing $0.27 \mathrm{mM}$ EDTA and applied to 96-well MicroFluor microtiter plates (ThermoLabsystems) for overnight incubation at $4^{\circ} \mathrm{C}(7)$. Samples were then incubated with isotype control Ab or EO6 (provided by J. L. Witztum, UCSD, San Diego, California, USA) for 2 hours at room temperature, followed by a goat-anti-mouse IgMAP-labeled secondary antibody (at 1:35,000; Sigma-Aldrich). For development, $25 \mu \mathrm{l}$ of $33 \%$ LumiPhos Plus solution (Lumigen) were added and light emissions were measured as RLU on a WALLAC VIKTOR II luminometer (PerkinElmer).

Bone marrow transplantation. Recipient C57BL/6 bone marrow was ablated with a single dose of radiation ( 9 Gy) using a Cobalt 60 irradiator (MDS Nordion) followed by injection of $2 \times 10^{6} \mathrm{C} 57 \mathrm{BL} / 6$ or Wave $1^{-1-}$ bone marrow cells via the retroorbital sinus as described (66). To verify lethal irradiation, 1 mouse from each group (WT or Wave1 $1^{-/-}$ recipients) did not receive bone marrow and was followed over approximately 10 days, after which all of them succumbed. After 9 weeks, successful reconstitution of donor peritoneal macrophages was verified by checking for WAVE1 transcripts in freshly isolated peritoneal macrophages of $n=3$ mice/group.

Confocal microscopy. Cytoskeletal staining was performed with Alexa Fluor 488-labeled phalloidin (Invitrogen). Blocking of unspecific background was done with PBS/1\% BSA for 30 minutes. Propidium iodide (PI) (Sigma-Aldrich) in the presence of $0.1 \%$ Triton $\mathrm{X}-100$ was used for nuclear staining. Cells were visualized using a LSM 510 Confocal Laserscanning microscope (Zeiss) with a $488 \mathrm{~nm}$ and $543 \mathrm{~nm}$ excitation line. Incubations with carrier, DMPC, or OxPAPC $(10 \mu \mathrm{g} / \mathrm{ml})$ were performed for 30 minutes; pretreatment with cytochalasin D, H89, PKA inhibitor amide ${ }_{14-22}$ (Calbiochem), and Ht-31 (Promega) was performed as indicated. Slides were mounted in Fluoprep (BioMerieux). Images were generated with a Zeiss 40 Neofluor $(\times 40 / 1,30$ oil) objective at room temperature. LSM510 Basis Software Release 3.0 (Service Pack 3.2) was used as acquisition software. For automated cell shape analysis, confocal microscopy images were generated as described above and 4 randomly selected microscope field images were generated for each condition. The images were split into green and blue channels (Alexa Fluor 488-labeled phalloidin [Invitrogen] and DAPI [Sigma-Aldrich], respectively), and the CellProfiler cell image analysis software (http:// www.cellprofiler.org) (67) was programmed to (a) load single images into the pipeline and identify primary objects (nuclei) using the Otsu Adaptative method with 2-class thresholding (threshold range 0.1-1), minimized weighted variance, and shape method to distinguish clumped objects; (b) identify secondary objects (cytoplasm), based on the previously identified primary objects (nuclei), using the propagation method with 2-class Otsu global thresholding (automatically calculated threshold range) and minimized weighted variance; and (c) generate tables containing cell counts, area, form factor, and perimeter measurements. Primary objects detected at the image border were excluded from the analysis.

PKA kinase assay. PKA kinase assay (PepTag; Promega) was performed according to the manufacturer's instructions. Briefly, RAW 264.7 cells were plated at a density of $1 \times 10^{7}$, treated as indicated, and then scratched off in PKA extraction buffer, homogenized using a 25 -gauge needle, and centrifuged for 5 minutes at $20,000 \mathrm{~g}$. Controls and sample reaction were prepared according to the manufacturer's instructions. After adjustment of protein contents, equal amounts were loaded on a $0.8 \%$ agarose gel, and chemiluminescence was recorded with a Bio-Rad UV-transilluminator.

Generation of PKAc and WAVE1 shRNA cell lines. PKAc and WAVE1 gene silencing was carried out by designing short hairpins using the siRNA target designer (Promega,) to nucleotide regions 930-948 (PKAc; GenBank NM_008854) and 219-237 (WAVE1; GenBank NM_031877). As a control, scrambled nucleotide sequences comprising the shRNA to each transcript were used (Supplemental Table 1). Nucleotides were annealed and ligated into the PstI site of the psiSTRIKE vector (Promega), and plasmids were transformed into competent DH5- $\alpha$ cells. Purified recombinant DNA (Promega Maxiprep kit) $(2 \mu \mathrm{g})$ was transfected into $2 \times 10^{6}$ RAW 264.7 cells using the Amaxa Cell Line Kit $\mathrm{V}$ (Amaxa). Transfected cells were selected with $7 \mu \mathrm{g} / \mathrm{ml}$ puromycin and stable cell lines generated.

Western blotting. Macrophages were washed and lysed as described (68), and $25 \mu \mathrm{g}$ of supernatant was separated by electrophoresis on a $10 \%$ SDS polyacrylamide gel. Following electrophoresis, the gels were transferred to PVDF membranes. Antibodies specific for PKAca (Santa Cruz Biotechnology Inc.) and WAVE1 (Sigma-Aldrich) were used at a dilution of 1:1000 and $\beta$-actin antibody (Sigma-Aldrich) at 1:500. Immunoreactive proteins were detected by the enhanced chemiluminescent protocol (GE Healthcare).

Evaluation of $m R N A$ expression in peritoneal macrophages. QIAGEN's RNEasy kit was used for RNA extraction, which included a DNase step, and cDNA was converted using the Superscript III first-strand synthesis system as described by the supplier (Invitrogen). RT-PCR was conducted according to the LightCycler FastStart DNA MasterPLUS SYBR Green I system using the Roche Light Cycler II sequence detector (Roche Applied Science). Sequences are listed in Supplemental Table 2.

Harvest and handling of PDF. In patients undergoing automated peritoneal dialysis, effluent samples were taken from a single long dwell performed with a conventional icodextrin-containing peritoneal dialysis solution (Extraneal; Baxter Healthcare). Dwell time was at least 14 hours. Informed consent was obtained from peritoneal dialysis patients before collection of effluent samples. PDF from these patients was depleted of IgG by repetitive incubation of $5 \mathrm{ml}$ PDF with $300 \mu \mathrm{l}$ protein G sepharose beads (GE Healthcare). Lipids were extracted by separating the PDF into 2 phases using chloroform/methanol, a method previously used to extract plasma lipids (www.cyberlipid.org/extract/extr0006.htm\#6). Briefly, $3.75 \mathrm{ml}$ of $0.5 \mathrm{M} \mathrm{KH}_{2} \mathrm{PO}_{4}$ was added to $2.5 \mathrm{ml}$ of PDF, followed by $18.75 \mathrm{ml}$ of chloroform and $6.25 \mathrm{ml}$ of methanol. This mix was then vortexed for 2 minutes and centrifuged at $440 \mathrm{~g}$ for 5 minutes to separate the upper aqueous phase from the lower lipid phase. 
Statistics. Data are presented as mean \pm SEM. Comparisons between groups were assessed using either the Mann-Whitney $U$ test or ANOVA followed by Bonferroni's multiple comparisons analysis, where appropriate. Survival data were analyzed by the Gehan-Breslow-Wilcoxon test using GraphPad Prism Software. $P<0.05$ was considered significant.

Study approval. The local animal care committee of the Medical University of Vienna and Ministry of Sciences approved all animal experiments. Patients gave informed consent.

\section{Acknowledgments}

We are grateful to Harald Höger for excellent support in mouse breeding, Rita Böhs for animal care, and Edith Pfeiffer for assistance with irradiation. We thank Marion Gröger for technical advice and Julia Schatz for excellent graphical assistance. This work was supported by a grant from the Austrian Science Fund (FWF-P18232-B11 to S. Knapp and V.N. Bochkov). J.D. Scott was supported in part by NIH grant DK54441.
Received for publication December 26, 2012, and accepted in revised form May 2, 2013.

Address correspondence to: Sylvia Knapp, CeMM - Center for Molecular Medicine of the Austrian Academy of Sciences, and Department of Medicine I, Laboratory of Infection Biology, Medical University Vienna, Waehringer Guertel 18-20, 1090 Vienna, Austria. Phone: 43.1.40400.5139; Fax: 43.1.40400.5167; E-mail: sylvia.knapp@meduniwien.ac.at.

Ulrich Matt's present address is: Division of Infectious Diseases and Hospital Epidemiology, University Hospital Zurich, University of Zurich, Switzerland.

Tanja Furtner's present address is: Institute of Molecular Regenerative Medicine, Paracelsus Medical University, Salzburg, Austria.
1. Hampton MB, Kettle AJ, Winterbourn CC. Inside the neutrophil phagosome: oxidants, myeloperoxidase, and bacterial killing. Blood. 1998;92(9):3007-3017.

2. Miller YI, et al. Oxidation-specific epitopes are danger-associated molecular patterns recognized by pattern recognition receptors of innate immunity. Circ Res. 2011;108(2):235-248.

3. Binder CJ, et al. Innate and acquired immunity in atherogenesis. Nat Med. 2002;8(11):1218-1226.

4. Hansson GK, Libby P. The immune response in atherosclerosis: a double-edged sword. Nat Rev Immunol. 2006;6(7):508-519.

5. Yoshimi N, et al. Oxidized phosphatidylcholine in alveolar macrophages in idiopathic interstitial pneumonias. Lung. 2005;183(2):109-121.

6. Nakamura T, Henson PM, Murphy RC. Occurrence of oxidized metabolites of arachidonic acid esterified to phospholipids in murine lung tissue. Anal Biochem. 1998;262(1):23-32.

7. Imai $Y$, et al. Identification of oxidative stress and Toll-like receptor 4 signaling as a key pathway of acute lung injury. Cell. 2008;133(2):235-249.

8. Matt U, et al. Bbeta(15-42) protects against acidinduced acute lung injury and secondary pseudomonas pneumonia in vivo. Am J Respir Crit Care Med. 2009;180(12):1208-1217.

9. Newcombe J, Li H, Cuzner ML. Low density lipoprotein uptake by macrophages in multiple sclerosis plaques: implications for pathogenesis. Neuro pathol Appl Neurobiol. 1994;20(2):152-162.

10. Dei $\mathrm{R}$, et al. Lipid peroxidation and advanced glycation end products in the brain in normal aging and in Alzheimer's disease. Acta Neuropathol. 2002;104(2):113-122

11. Bochkov VN, Kadl A, Huber J, Gruber F, Binder BR, Leitinger N. Protective role of phospholipid oxidation products in endotoxin-induced tissue damage. Nature. 2002;419(6902):77-81.

12. Knapp S, Matt U, Leitinger N, van der Poll T. Oxidized phospholipids inhibit phagocytosis and impair outcome in gram-negative sepsis in vivo. J Immunol. 2007;178(2):993-1001.

13. Cruz D, et al. Host-derived oxidized phospholipids and HDL regulate innate immunity in human leprosy. J Clin Invest. 2008;118(8):2917-2928.

14. Holzheimer RG, Muhrer KH, L'Allemand N, Schmidt T, Henneking K. Intraabdominal infections: classification, mortality, scoring and pathophysiology. Infection. 1991;19(6):447-452.

15. Wickel DJ, Cheadle WG, Mercer-Jones MA, Garrison $\mathrm{RN}$. Poor outcome from peritonitis is caused by disease acuity and organ failure, not recurrent peritoneal infection. Ann Surg. 1997;225(6):744-753

16. Pinheiro da Silva F, et al. CD16 promotes Escherichia coli sepsis through an $\mathrm{FcR}$ gamma inhibitory pathway that prevents phagocytosis and facilitates inflammation. Nat Med. 2007;13(11):1368-1374.

17. Underhill DM, Ozinsky A. Phagocytosis of microbes: complexity in action. Annu Rev Immunol. 2002;20:825-852.

18. Takenawa T, Suetsugu S. The WASP-WAVE protein network: connecting the membrane to the cytoskeleton. Nat Rev Mol Cell Biol. 2007;8(1):37-48.

19. Westphal RS, Soderling SH, Alto NM, Langeberg LK, Scott JD. Scar/WAVE-1, a Wiskott-Aldrich syndrome protein, assembles an actin-associated multi-kinase scaffold. Embo J. 2000;19(17):4589-4600.

20. Dinh H, Scholz GM, Hamilton JA. Regulation of WAVE1 expression in macrophages at multiple levels. J Lenkoc Biol. 2008;84(6):1483-1491.

21. Friedman P, Horkko S, Steinberg D, Witztum JL, Dennis EA. Correlation of antiphospholipid antibody recognition with the structure of synthetic oxidized phospholipids. Importance of Schiff base formation and aldol condensation. J Biol Chem. 2002;277(9):7010-7020.

22. Kaksonen M, Toret CP, Drubin DG. Harnessing actin dynamics for clathrin-mediated endocytosis. Nat Rev Mol Cell Biol. 2006;7(6):404-414.

23. Miller YI, Worrall DS, Funk CD, Feramisco JR, Witztum JL. Actin polymerization in macrophages in response to oxidized LDL and apoptotic cells: role of 12/15-lipoxygenase and phosphoinositide 3-kinase. Mol Biol Cell. 2003;14(10):4196-4206.

24. Park YM, Febbraio M, Silverstein RL. CD36 modulates migration of mouse and human macrophages in response to oxidized LDL and may contribute to macrophage trapping in the arterial intima. J Clin Invest. 2009;119(1):136-145.

25. Boullier A, et al. The binding of oxidized low density lipoprotein to mouse CD36 is mediated in part by oxidized phospholipids that are associated with both the lipid and protein moieties of the lipoprotein. J Biol Chem. 2000;275(13):9163-9169.

26. Li R, et al. Identification of prostaglandin E2 receptor subtype 2 as a receptor activated by OxPAPC. Circ Res. 2006;98(5):642-650.

27. Birukova AA, Chatchavalvanich S, Oskolkova O, Bochkov VN, Birukov KG. Signaling pathways involved in OxPAPC-induced pulmonary endothelial barrier protection. Microvasc Res. 2007;73(3):173-181.

28. Walton KA, et al. Specific phospholipid oxidation products inhibit ligand activation of toll-like receptors 4 and 2. Arterioscler Thromb Vasc Biol. 2003;23(7):1197-1203.

29. Seyerl $M$, et al. Oxidized phospholipids induce anergy in human peripheral blood T cells. Eur J Immunol. 2008;38(3):778-787.

30. Levitan I, Gooch KJ. Lipid rafts in membrane- cytoskeleton interactions and control of cellular biomechanics: actions of oxLDL. Antioxid Redox Signal. 2007;9(9):1519-1534.

31. Birukov KG, et al. Epoxycyclopentenone-containing oxidized phospholipids restore endothelial barrier function via Cdc42 and Rac. Circ Res. 2004; 95(9):892-901.

32. Bryn T, Mahic M, Enserink JM, Schwede F, Aandahl EM, Tasken K. The cyclic AMP-Epac1-Rap1 pathway is dissociated from regulation of effector functions in monocytes but acquires immunoregulatory function in mature macrophages. J Immunol. 2006;176(12):7361-7370.

33. Scott JD, Fischer EH, Demaille JG, Krebs EG. Identification of an inhibitory region of the heat-stable protein inhibitor of the cAMP-dependent protein kinase. Proc Natl Acad Sci US A. 1985;82(13):4379-4383.

34. Scott JD, Glaccum MB, Fischer EH, Krebs EG. Primary-structure requirements for inhibition by the heat-stable inhibitor of the cAMP-dependent protein kinase. Proc Natl Acad Sci U S A. 1986;83(6):1613-1616.

35. Lochner A, Moolman JA. The many faces of H89: a review. Cardiovasc Drug Rev. 2006;24(3-4):261-274.

36. Cheng HC, et al. A potent synthetic peptide inhibitor of the cAMP-dependent protein kinase. J Biol Chem. 1986;261(3):989-992.

37. Kim C, Xuong NH, Taylor SS. Crystal structure of a complex between the catalytic and regulatory (RIalpha) subunits of PKA. Science. 2005; 307(5710):690-696

38. Tasken K, Aandahl EM. Localized effects of cAMP mediated by distinct routes of protein kinase $\mathrm{A}$. Physiol Rev. 2004;84(1):137-167.

39. Scott JD, Pawson T. Cell signaling in space and time: where proteins come together and when they're apart. Science. 2009;326(5957):1220-1224.

40. Carr DW, Hausken ZE, Fraser ID, Stofko-Hahn RE, Scott JD. Association of the type II cAMPdependent protein kinase with a human thyroid RII-anchoring protein. Cloning and characterization of the RII-binding domain. J Biol Chem. 1992;267(19):13376-13382.

41. Soderling SH, et al. Loss of WAVE-1 causes sensorimotor retardation and reduced learning and memory in mice. Proc Natl Acad Sci U S A. 2003;100(4):1723-1728.

42. Murch AR, Grounds MD, Papadimitriou JM. Improved chimaeric mouse model confirms that resident peritoneal macrophages are derived solely from bone marrow precursors. J Pathol. 1984;144(2):81-87.

43. Krediet RT, Balafa O. Cardiovascular risk in the peritoneal dialysis patient. Nat Rev Nephrol. 2010;6(8):451-460. 
44. Himmelfarb J, Hakim RM. Oxidative stress in uremia. Curr Opin Nephrol Hypertens. 2003;12(6):593-598.

45. Oberg BP, et al. Increased prevalence of oxidant stress and inflammation in patients with moderate to severe chronic kidney disease. Kidney Int. 2004;65(3):1009-1016.

46. Troidle L, Finkelstein F. Treatment and outcome of CPD-associated peritonitis. Ann Clin Microbiol Antimicrob. 2006;5:6.

47. Sarnak MJ, Jaber BL. Mortality caused by sepsis in patients with end-stage renal disease compared with the general population. Kidney Int. 2000;58(4):1758-1764.

48. Palinski W, et al. Cloning of monoclonal autoantibodies to epitopes of oxidized lipoproteins from apolipoprotein E-deficient mice. Demonstration of epitopes of oxidized low density lipoprotein in human plasma. J Clin Invest. 1996;98(3):800-814.

49. Chang MK, et al. Monoclonal antibodies against oxidized low-density lipoprotein bind to apoptotic cells and inhibit their phagocytosis by elicited macrophages: evidence that oxidation-specific epitopes mediate macrophage recognition. Proc Natl Acad Sci US A. 1999;96(11):6353-6358.

50. Soderling $\mathrm{SH}$, et al. A WAVE-1 and WRP signaling complex regulates spine density, synaptic plasticity, and memory. J Neurosci. 2007;27(2):355-365.

51. Kim Y, et al. Phosphorylation of WAVE1 regulates actin polymerization and dendritic spine morphology. Nature. 2006;442(7104):814-817.

52. Cameron JS. Host defences in continuous ambula- tory peritoneal dialysis and the genesis of peritonitis. Pediatr Nephrol. 1995;9(5):647-662.

53. Kato $S$, et al. Aspects of immune dysfunction in end-stage renal disease. Clin J Am Soc Nephrol. 2008;3(5):1526-1533.

54. Muller LM, et al. Increased risk of common infections in patients with type 1 and type 2 diabetes mellitus. Clin Infect Dis. 2005;41(3):281-288.

55 . Jager KJ, et al. Cardiovascular and non-cardiovascular mortality in dialysis patients: where is the link? Kidney Int Sup. 2011;1:21-23.

56. Latcha S, Hong S, Gibbons N, Kohn N, Mattana J. Relationship between dialysate oxidized protein and peritoneal membrane transport properties in patients on peritoneal dialysis. Nephrol Dial Transplant. 2008;23(10):3295-3301.

57. Posthuma N, ter Wee P, Donker AJ, Dekker HA, Oe PL, Verbrugh HA. Peritoneal defense using icodextrin or glucose for daytime dwell in CCPD patients. Perit Dial Int. 1999;19(4):334-342.

58. Poyrazoglu HM, Dusunsel R, Patiroglu T, Gunduz Z, Utas C, Gunes T. Humoral immunity and frequency of peritonitis in chronic peritoneal dialysis patients. Pediatr Nephrol. 2002;17(2):85-90.

59. Horkko S, et al. Monoclonal autoantibodies specific for oxidized phospholipids or oxidized phospholipid-protein adducts inhibit macrophage uptake of oxidized low-density lipoproteins. J Clin Invest. 1999;103(1):117-128.

60. Subbanagounder G, et al. Epoxyisoprostane and epoxycyclopentenone phospholipids regulate monocyte chemotactic protein-1 and interleukin-8 synthesis. Formation of these oxidized phospholipids in response to interleukin-1 $\beta$. J Biol Chem. 2002;277(9):7271-7281.

61. Maier S, et al. Cecal ligation and puncture versus colon ascendens stent peritonitis: two distinct animal models for polymicrobial sepsis. Shock. 2004;21(6):505-511.

62. Bosmann M, Russkamp NF, Patel VR, Zetoune FS, Sarma JV, Ward PA. The outcome of polymicrobial sepsis is independent of T and B cells. Shock. 2011;36(4):396-401.

63. Reim D, Westenfelder K, Kaiser-Moore S, Schlautkotter S, Holzmann B, Weighardt H. Role of T cells for cytokine production and outcome in a model of acute septic peritonitis. Shock. 2009; 31(3):245-250.

64. Riedemann NC, Guo RF, Ward PA. The enigma of sepsis. J Clin Invest. 2003;112(4):460-467.

65 . Hoebe K, et al. CD36 is a sensor of diacylglycerides. Nature. 2005;433(7025):523-527.

66. Pawlinski R, et al. Role of tissue factor and protease-activated receptors in a mouse model of endotoxemia. Blood. 2004;103(4):1342-1347.

67. Carpenter AE, et al. CellProfiler: image analysis software for identifying and quantifying cell phenotypes. Genome Biol. 2006;7(10):R100.

68. Lagler $\mathrm{H}$, et al. TREM-1 activation alters the dynamics of pulmonary IRAK-M expression in vivo and improves host defense during pneumococcal pneumonia. J Immunol. 2009;183(3):2027-2036. 